\title{
Acute Myocardial Infarction Due to Left Main Coronary Artery( LMCA) Occlusion in a Teenage Girl
}

\author{
Pritam Kitey $^{1}$, Vikas Kataria ${ }^{1},{\text { Amitabh } \text { Yaduvanshi }^{1} \text {, and MOHAN NAIR }}^{1}$ \\ ${ }^{1}$ Holy Family Hospital
}

July 7, 2020

\begin{abstract}
Acute myocardial infarction (AMI) in teenagers and young adults is an uncommon phenomenon. The etiology and pathophysiology of AMI in this age group is different than the AMI in older population. We report an interesting and rare case of AMI in a teenage girl due to occlusion of LMCA
\end{abstract}

\section{Abbreviations}

AMI- Acute Myocardial Infarction

ANA- Anti Nuclear Antibody

APLS - Anti Phospholipid Syndrome

CAG - Coronary Angiography

CAD - Coronary Artery Disease

ECG - Electrocardiography

LMCA- Left Main Coronary Artery

LAD- Left Anterior Descending

LCx- Left Circumflex

RCA- Right Coronary Artery

\section{Introduction}

Acute myocardial infarction (AMI) in young patients aged [?] 35 years is an uncommon phenomenon and estimated to account for less than $2 \%$ of all $\mathrm{AMI}^{1}$. AMI in teenagers is even more rare ${ }^{2}$. The pathophysiology of AMI in this age group is varied but not usually due to atherosclerotic plaque rupture. Appropriate treatment has to be adapted from adult management protocols, as there are no controlled trials to guide early treatment of myocardial infarction in this age group. We report an interesting and rare case of AMI in a teenage girl where the etiology was most likely to be atherosclerosis.

\section{Case Report}

A 19-year-old non-obese $\left(\mathrm{BMI}=20.2 \mathrm{~kg} / \mathrm{m}^{2}\right)$ girl presented with chest pain associated with nausea and vomiting of 1 hour duration. She was pale and sweaty with a tachycardia of 110 beats/min. Her systolic blood pressure was $60 \mathrm{~mm} \mathrm{Hg}$, which responded to intravenous fluid therapy and inotropic support. There was no history of smoking, drug or analgesic abuse, previous episodes of arterial or venous thrombosis. There is no significant medical or surgical history and she was not on any medicine (including herbal). There was no 
peripheral edema and all peripheral pulses were present. Her heart sounds were normal and there were no signs of heart failure. She had never previously experienced chest pain at rest or on exertion.

Electrocardiography (ECG) showed sinus rhythm with ST elevation in I, avL, V4-V6 with reciprocal changes in inferior leads (Figure 1). Transthoracic echocardiography showed anterior wall to be hypokinetic with left ventricular ejection fraction (LVEF) $40 \%$ (Figure 2). Urgent coronary angiography (CAG) was done which revealed ostial 99\% lesion in left main coronary artery (LMCA) with distal TIMI I flow (figure 3a). Intracoronary bolus of nitroglycerine $50 \mu \mathrm{g}$ did not improve coronary blood flow. Immediate angioplasty (door-to-balloon time $=25$ minutes) and stenting to LMCA was done and TIMI III flow achieved (figure 3b). Post angioplasty, imaging showed plaque in left anterior descending (LAD) artery, normal circumflex (Lcx) and right coronary artery (RCA). There was no collateral blood flow to the left circulations from RCA. Optical coherence tomography (OCT) was done which confirmed well expanded stent and presence of atherosclerotic plaque in mid LAD. We were able to finish the procedure without any IABP support.

Inotropic support was gradually withdrawn as BP improved after restoration of coronary blood flow. During hospital stay she was evaluated for possible etiology of AMI. All laboratory parameters were within normal limits. Hemoglobin $9.8 \mathrm{gm} / \mathrm{dl}(\mathrm{n}=12-16)$, serum creatinine $0.84 \mathrm{mg} / \mathrm{dl}(\mathrm{n}=0.5-1.2 \mathrm{mg} / \mathrm{dl})$, Homocysteine 7.53 umol/L ( $\mathrm{n}=4.4-13.5)$, phospholipid antibody 0.71GPL u/l $(<10)$, Total Cholesterol (TC) $107 \mathrm{mg} / \mathrm{dl}(0-200)$, Triglyceride(TG) 66mg/dl (0-150), Low Density Lipoprotein (LDL) $62 \mathrm{mg} / \mathrm{dl}$ (0-100), serum cortisol 34.23, Thyroid Stimulating Hormone (TSH) $2.34 \mu \mathrm{IU} / \mathrm{ml}(0.4-4.2)$. She was evaluated for the cause of anemia by hematologist. The blood picture was suggestive of Nutritional Iron Deficiency Anemia. She was discharged on oral Iron therapy.

There was no coronary artery anomaly. CT aortogram did not reveal any disease in the aorta or its major branches. Her father had AMI at the age of 39 years and medical records revealed coronary atherosclerosis to be the cause of AMI. The patient was discharged on 4th day on dual antiplatelet therapy, beta blocker, statin. Levels of protein $\mathrm{C}$, protein $\mathrm{S}$ and antithrombin III were performed after 8 weeks of AMI and were found to be normal. All other laboratory parameters Anti Phospholipid Antibody (APLA) panel (Lupus anticoagulant, anticardiolipin Antibody, Anti beta 2 glycoprotein), Antinuclear Antibody (ANA) were normal. The indicators pointing towards atherosclerosis as the possible etiology were a) plaque in mid LAD, b) very strong family history of coronary artery disease (CAD) and c) absence of any biochemical or imaging abnormality that suggested an alternative etiology.

\section{Discussion:}

Acute MI in the teenage years of life is a rare phenomenon. Data on AMI in young adults is limited to few case reports ${ }^{3}$. In these cases the etiology of MI was either hypercoagulable state, autoimmune disease or coronary spasm, dissection or coronary embolization ${ }^{2-8}$.

S. Osula reported a case of 16 year old boy presenting as AMI,but the etiology was hypercoagulable state resulting from nephrotic syndrome ${ }^{3}$. A case of 23 years old male presenting with AMI was reported by Mustafa cetin. Etiology of AMI in this case was Behcet's disease ${ }^{4}$. Vale PR reported a case of spontaneous coronary artery dissection involving the left circumflex artery in a young adult male presenting with a myocardial infarction after exercise ${ }^{6}$. L Abid et al found primary antiphospholipid syndrome (APLS) as a causative factor of AMI in two young adults ${ }^{7}$. Coronary artery embolism was the cause of acute MI in the setting of an existing ASD in a case reported by Jonathan Kei ${ }^{2}$. A case of AMI in a boy of age similar to our patient was reported by $S$. Ouali et al. In this case, coronary artery disease was associated with aneurysmal dilatation in the carotid, vertebral and right renal arteries and it was diagnosed as aortoarteritis. ${ }^{9}$

None of these case reports attributed AMI to atherosclerosis. In the present case AMI occurred in a teenaged girl, who is non-smoker, non drug abuser, no evidence of hypercoagulability, autoimmune disease and coronary anomalies. Absence of these factors, a very strong family history of CAD and presence of plaque in LAD were strong indicators towards atherosclerosis as the cause of AMI in this patient.

To the best of our knowledge, this is the first case report of AMI in a teenage girl occurring because of 
atherosclerosis. The data regarding the incidence of atherosclerosis CAD in this age is limited but the reported incidence (15-19 years) is $2 \%$ (all males) (8). There is no reports of atherosclerosis causing AMI in teenaged girl.

\section{Conclusion:}

Acute MI in the teenage years of life is a rare phenomenon. The etiology and pathophysiology of AMI in this age group is completely different from the AMI in older population. One should keep atherosclerosis as an etiological differential diagnosis of AMI even in teenagers.

\section{Author Contribution:}

Pritam Kitey: Helped in clinical management of the case and manuscript preparation

Vikas Kataria: Contributed in clinical and interventional management of the case and in manuscript writing.

Amitabh Yaduvanshi: Contributed in clinical and interventional management of the case

Mohan Nair: Contributed in clinical and interventional management of the case, Manuscript writing and overall supervision.

\section{References:}

1) Fournier JA, Cabezon S, Cayuela A, Ballesteros SM, Cortacero JA, Diaz De. Long-term prognosis of patients having acute myocardial infarction when $</=40$ years of age. Am J Cardiol. 2004;94(8):989.

2. Kei J, Avilla JK, Cavendish JJ. Rare case of myocardial infarction in a 19-year-old caused by a paradoxical coronary artery embolism. Perm J. 2015;19:e107-9

3.Osula S, Bell GM, Hornung RS Acute myocardial infarction in young adults: causes and management Postgraduate Medical Journal 2002;78:27-30.

4.Cetin M, Ozturk U, Cakici M, et alAcute inferior myocardial infarction in a young male patient associated with Behcet's disease and sildenafilCase Reports 2014;2014:bcr2013201189.

5.Jouhikainen T, Pohjola-Sintonen S, Stephansson E. Lupus anticoagulant and cardiac manifestations in systemic lupus erythematosus. Lupus1994;3:167.

6.Vale PR, Baron DW. Coronary artery stenting for spontaneous coronary artery dissection: a case report and review of the literature. Cathet Cardiovasc Diagn1998;45:280-6.

7. Abid L, Frikha F, Bahloul Z, Kammoun S. Acute myocardial infarction in young adults with antiphospholipid syndrome: report of two cases and literature review. Pan Afr Med J. 2011;8:13. doi:10.4314/pamj.v8i1.71062

8. Mc Gill HC, McMahan CA, Zieske AW, et al. Association of coronary heart disease risk factors with microscopic qualities of coronary atherosclerosis in youth. Circulation2000;102:375.

9. Ouali S, Kacem S, Ben Fradj F, et al. Takayasu arteritis with coronary aneurysms causing acute myocardial infarction in a young man. Tex Heart Inst J. 2011;38(2):183-186.

\section{Figure Legends}

Figure 1 : ECG: ST elevation in leads I,V1-V4 with ST depression in leads II.III.

(avL,avF, avR are not shown)

Figure 2: Echocardiography showing akinesia of LV apex

Figure 3 a: Still image coronary angiogram showing LMCA ostial $99 \%$ stenosis

with distal total occlusion (LMCA- Left Main Coronary Artery). 
Figure 3b : Still image from post angioplasty coronary angiogram shows normal caliber LMCA with TIMI III flow distally (LMCA- Left Main Coronary Artery)
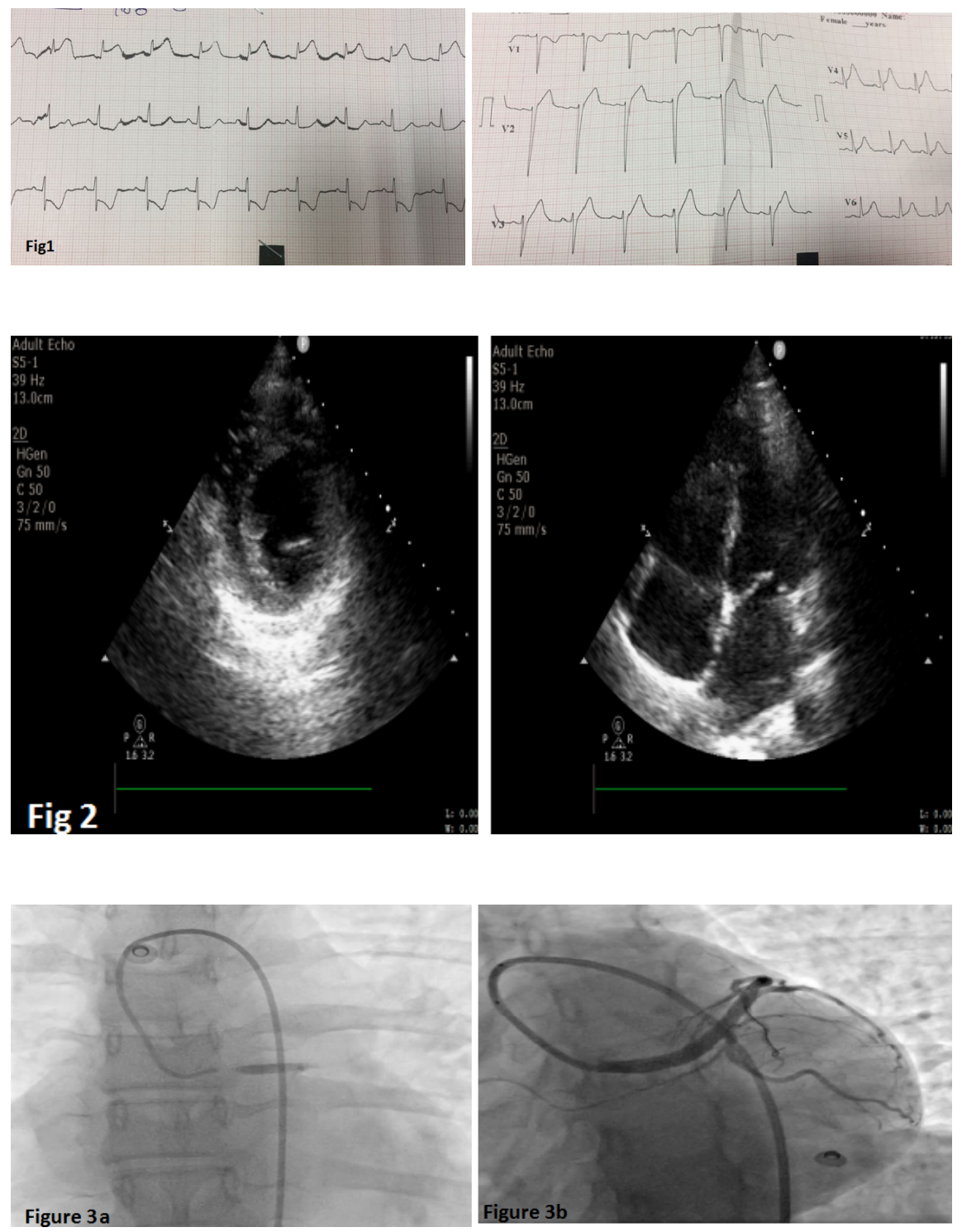